Pure and Applied Mathematics Quarterly

Volume 2, Number 1

(Special Issue: In honor of

John H. Coates, Part 1 of 2)

$111-133,2006$

\title{
Derivatives of Modular Forms of Negative Weight
}

\author{
Daniel Bump and Young-Ju Choie \\ Dedicated to John Coates
}

Let $G=\operatorname{Sp}(2 n, \mathbb{R})$, and let $\Gamma$ be a discrete subgroup. Let $\chi: \Gamma \longrightarrow \mathbb{C}$ be a function such that when $Z \in \mathcal{H}_{n}$, the Siegel space of genus $n$, the multiplier system

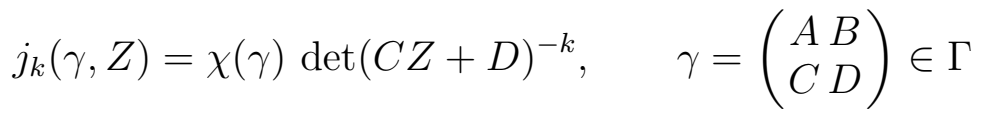

satisfies the cocycle condition

$$
j_{k}\left(\gamma \gamma^{\prime}, Z\right)=j_{k}\left(\gamma, \gamma^{\prime} Z\right) j_{k}\left(\gamma^{\prime}, Z\right) .
$$

It is important for us to allow $k \in \frac{1}{2} \mathbb{Z}$. If $k$ is half-integral, then $\operatorname{det}(C Z+$ $D)^{-k}$ involves the choice of a branch of square root, but this is unimportant since a different choice of branch can be compensated for in the choice of the function $\chi$.

Let $M_{k}(\Gamma, \chi)$ be the space of meromorphic functions $f$ on $\mathcal{H}_{n}$ such that

$$
f(Z)=j_{k}(\gamma, Z) f(\gamma Z) .
$$

Let $Z=\left(Z_{i j}\right) \in \mathcal{H}_{n}$, and let $\partial_{i j}$ be the differential operator defined by

$$
\partial_{i j}=\left(\frac{1+\delta_{i j}}{2}\right) \frac{\partial}{\partial Z_{i j}} .
$$

(Note that $Z_{i j}$ and $Z_{j i}$ are the same variable.) Let

$$
\mathbb{D}_{n}=\operatorname{det}\left(\partial_{i j}\right) \text {. }
$$

Received July 22, 2005. 
Conjecture 1. Let $r \geqslant 0$ be an integer. If $f \in M_{-r+\frac{n-1}{2}}(\Gamma, \chi)$, then the derivative $\mathbb{D}_{n}^{r+1} f \in M_{r+2+\frac{n-1}{2}}(\Gamma, \chi)$.

If $n=1$, Conjecture 1 can be proved as follows. Let $\mathcal{H}=\mathcal{H}_{n}$ be the usual upper half plane. Let $S$ be a finite set of points in $\Gamma \backslash \mathcal{H}$, where a modular form is allowed to have poles. Let $\mathcal{H}_{S}$ be the set of all $z \in \mathcal{H}$ such that the image of $z$ in $\Gamma \backslash \mathcal{H}$ is not in $S$. Define

$$
\mathbb{D}=\frac{d}{d z}, \quad \partial_{k}=\frac{1}{2 \pi i}\left(\mathbb{D}-\frac{i k}{2 y}\right)
$$

In the notation of the next section, $\partial_{k}=-\frac{1}{4 \pi} \mathcal{R}_{k}$. The operator $\partial_{k}$ does not preserve holomorphicity but preserves the space $\mathcal{M}_{k}^{S}(\Gamma, \chi)$ of smooth functions $f: \mathcal{H}_{S} \longrightarrow \mathbb{C}$ such that

$$
f(z)=j_{k}(\gamma, z) f\left(\frac{a z+b}{c z+d}\right), \quad z \in \mathcal{H}_{S}, \quad g=\left(\begin{array}{l}
a b \\
c d
\end{array}\right) \in G .
$$

one proves by induction the identity of Bol [1]:

$$
\partial_{k}^{h}=\partial_{k+2 h-2} \circ \ldots \circ \partial_{k+2} \circ \partial_{k}=\left(-\frac{1}{4 \pi y}\right)^{h} \sum_{j=0}^{h}\left(\begin{array}{l}
h \\
j
\end{array}\right) \frac{\Gamma(h+k)}{\Gamma(j+k)}(2 i y \mathbb{D})^{j} .
$$

It is understood that the term is zero if $j+k$ is a nonpositive integer but $h+k$ is not, since then $\Gamma(j+k)^{-1}=0$ but $\Gamma(h+k)$ has no pole. In particular, there is only one nonzero term in

$$
\partial_{-r}^{r+1}=\left(-\frac{1}{4 \pi y}\right)^{r+1} \sum_{j=0}^{r+1}\left(\begin{array}{c}
r+1 \\
j
\end{array}\right) \frac{\Gamma(1)}{\Gamma(j-r)}(2 i y \mathbb{D})^{j}=\left(\frac{1}{2 \pi i} \mathbb{D}\right)^{r+1} .
$$

As a consequence $\mathbb{D}^{r+1}$ maps holomorphic functions in $\mathcal{M}_{-r}^{S}(\Gamma)$ into $\mathcal{M}_{r+2}^{S}(\Gamma)$, and if such a function is meromorphic on $\mathcal{H}$, so of course is $\mathbb{D}^{r+1} f$.

The purpose of this paper is to reveal some underlying representation theory behind Conjecture 1 and to prove it when $n \leqslant 2$. When $n=1$, the alternative proof that we will give below in Theorem 1 is different from the one just given using the inductive formula or the result of Bol [1], and reveals an underlying reason why the statement is true. We will see that given a form of negative (integral) weight for $\mathrm{SL}(2, \mathbb{R})$, we may construct an "automorphic representation" by transferring it to the group and considering the $(\mathfrak{g}, K)$-module that it generates. This representation is reducible but indecomposable, and it has a representation of the holomorphic discrete 
series as an irreducible quotient. The interesting feature is that it has two "holomorphic vectors" corresponding to $f$ and $f^{(r+1)}$.

We will formulate the purely representation-theoretic Conjecture 2 which implies Conjecture 1 , and prove it when $n=2$. When $n=2$, the modular form must be of half-integral weight, and so the representations we consider will be not of $\operatorname{Sp}(2 n, \mathbb{R})$, but of the metaplectic group.

If $n$ is even, Choie and Kim [4] used another very different method to prove a similar result, using the Fourier-Jacobi expansion and Bol's identity. This approach requires that the group be of a particular type; for example it could not work if $\Gamma$ is cocompact.

This work was partially supported by NSF Grant DMS-0354662 and KOSEF Grant R01-2003-00011596-0. We would like to thank the referee for a careful reading.

\section{THE CASE $n=1$}

To clarify the ideas we start with the case $n=1$. It will be noted that when $n$ is even, Conjecture 1 involves modular forms of half-integral weight $r+\frac{n-1}{2}$. Since in this case $n$ is odd, this does not apply here and there is no need to introduce the metaplectic group. We will suppress the character $\chi$, and also consider only modular forms which are holomorphic in $\mathcal{H}$. (If the weight is negative, such a function must have poles at the cusps of $\Gamma$.)

Let $G=\mathrm{SL}(2, \mathbb{R})$, and let $\Gamma$ be a discrete subgroup. Let $\mathcal{M}_{k}(\Gamma)$ be the space of smooth functions satisfying (3). They are not assumed to be holomorphic. The subspace of holomorphic functions will be denoted $M_{k}(\Gamma)$. We allow $k$ to be negative. Denote by $\mathcal{C}_{k}(\Gamma \backslash \mathcal{H})$ the space of smooth functions $f: \mathcal{H} \longrightarrow \mathbb{C}$ such that

$$
f(z)=\chi(\gamma)\left(\frac{c \bar{z}+d}{|c z+d|}\right)^{k} f\left(\frac{a z+b}{c z+d}\right), \quad z \in \mathcal{H}, \quad \gamma=\left(\begin{array}{ll}
a & b \\
c d
\end{array}\right) \in \Gamma
$$

Finally, let $\mathcal{C}_{k}(\Gamma \backslash G)$ be the space of smooth functions $f: G \longrightarrow \mathbb{C}$ such that $f(\gamma g)=f(g)$ for $\gamma \in \Gamma$ and $f\left(g \kappa_{\theta}\right)=e^{i k \theta} f(g)$, where

$$
\kappa_{\theta}=\left(\begin{array}{cc}
\cos \theta & \sin \theta \\
-\sin \theta & \cos \theta
\end{array}\right) .
$$

We have isomorphisms

$$
\mathcal{M}_{k}(\Gamma) \stackrel{y^{k / 2}}{\longrightarrow} \mathcal{C}_{k}(\Gamma \backslash \mathcal{H}) \stackrel{\sigma_{k}}{\longrightarrow} \mathcal{C}_{k}(\Gamma \backslash G)
$$


where $y^{k / 2}$ is just multiplication by $y^{k / 2}$ and $\sigma_{k}$ is defined by

$$
\sigma_{k}(f)(g)=\left(\left.f\right|_{k} g\right)(i)
$$

where

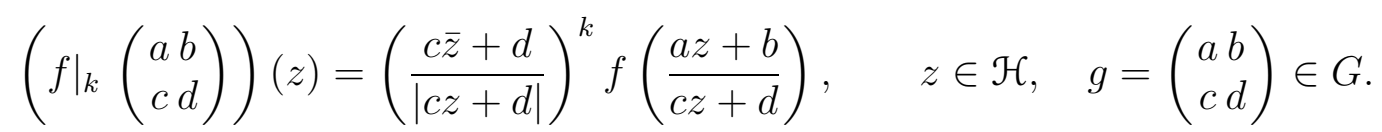

We have Maass operators (Maass [11]) on $\mathcal{C}_{k}(\Gamma \backslash \mathcal{H}$ ) defined by

$$
\begin{aligned}
& R_{k}=i y \frac{\partial}{\partial x}+y \frac{\partial}{\partial y}+\frac{k}{2}=(z-\bar{z}) \frac{\partial}{\partial z}+\frac{k}{2} \\
& L_{k}=-i y \frac{\partial}{\partial x}+y \frac{\partial}{\partial y}-\frac{k}{2}=(z-\bar{z}) \frac{\partial}{\partial \bar{z}}-\frac{k}{2},
\end{aligned}
$$

with

$$
\frac{\partial}{\partial z}=\frac{1}{2}\left(\frac{\partial}{\partial x}-i \frac{\partial}{\partial y}\right), \quad \frac{\partial}{\partial \bar{z}}=\frac{1}{2}\left(\frac{\partial}{\partial x}+i \frac{\partial}{\partial y}\right), \quad z=x+i y \in \mathcal{H} .
$$

Let $\mathfrak{g}$ be the Lie algebra of $G$, identified with the Lie algebra of $2 \times 2$ real matrices of trace zero. It acts on smooth functions as follows. If $X \in \mathfrak{g}$ and $f: G \longrightarrow \mathbb{C}$ is smooth then

$$
(X f)(g)=\left.\frac{d}{d t} f\left(g e^{i t X}\right)\right|_{t=0} .
$$

This action is extended to the complexification $\mathfrak{g}_{\mathbb{C}}$ and to the universal enveloping algebra $U\left(\mathfrak{g}_{\mathbb{C}}\right)$. Let

$$
R=\frac{1}{2}\left(\begin{array}{cc}
1 & i \\
i & -1
\end{array}\right), \quad L=\frac{1}{2}\left(\begin{array}{cc}
1 & -i \\
-i & -1
\end{array}\right), \quad H=-i\left(\begin{array}{cc}
0 & 1 \\
-1 & 0
\end{array}\right) \in \mathfrak{g}_{\mathbb{C}} .
$$

We have, in $\mathfrak{g}_{\mathbb{C}}$, the commutation relations

$$
[H, R]=2 R, \quad[H, L]=2 L, \quad[R, L]=H .
$$

Let

$$
-4 \Delta=H^{2}+2 R L+2 L R .
$$

This is the Casimir element, in the center of $U\left(\mathfrak{g}_{\mathbb{C}}\right)$. Then $\mathcal{C}_{k}(\Gamma \backslash G)$ is just the subspace of $C^{\infty}(\Gamma \backslash G)$ consisting $H$-eigenfunctions $f$ with $H f=k f$. Since $[R, L]=H$ we have

$$
-4 \Delta=H^{2}+2 H+4 L R=H^{2}-2 H+4 R L .
$$


We define operators $R_{k}, L_{k}$ and $\Delta_{k}$ on $\mathcal{C}_{k}(\Gamma \backslash G)$, and operators $\mathcal{R}_{k}, \mathcal{L}_{k}$ and $\triangle_{k}$ on $\mathcal{C}_{k}(\Gamma \backslash \mathcal{H})$ by asking that the following diagrams be commutative:

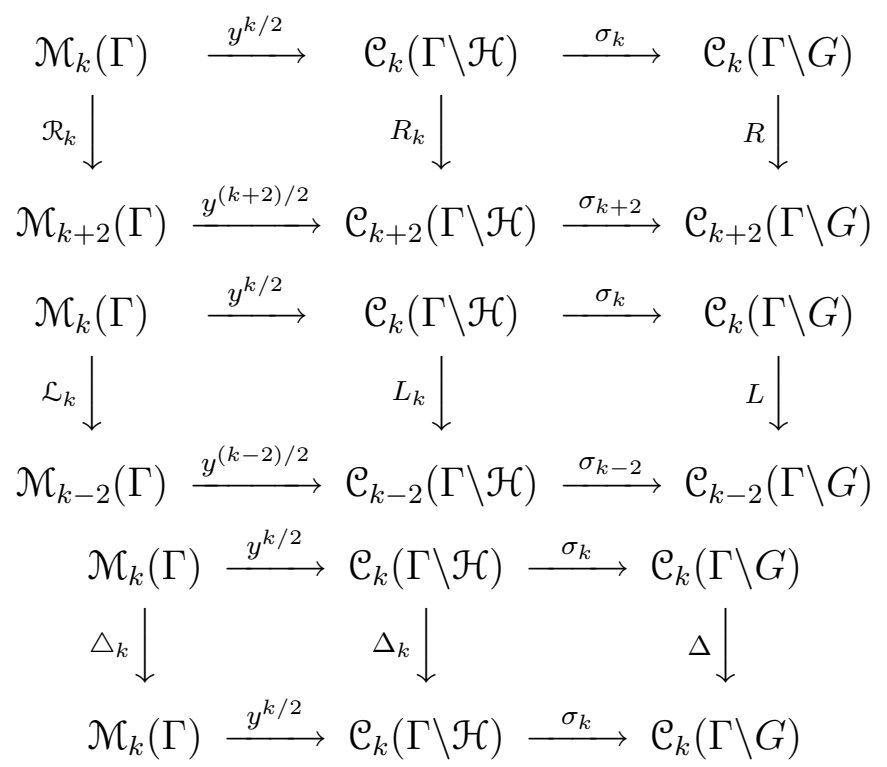

We have, in particular

$$
L_{k}=-2 i y \frac{\partial}{\partial \bar{z}}-\frac{k}{2}, \quad R_{k}=2 i y \frac{\partial}{\partial z}+\frac{k}{2}
$$

SO

$$
\begin{gathered}
\mathcal{R}_{k}=y^{-(k+2) / 2} R_{k} y^{k / 2}=2 i \frac{\partial}{\partial z}+\frac{k}{y} \\
\mathcal{L}_{k}=y^{-(k-2) / 2} L_{k} y^{k / 2}=2 i y^{2} \frac{\partial}{\partial \bar{z}}
\end{gathered}
$$

Thus, by the Cauchy-Riemann equations, $f \in \mathcal{M}_{k}(\Gamma)$ is holomorphic if and only if $\mathcal{L}_{k} f=0$, that is

$$
M_{k}(\Gamma)=\operatorname{ker}\left(\mathcal{L}_{k}\right) .
$$

Finally, we note that $\Delta$ and $R$ commute in $U\left(\mathfrak{g}_{\mathbb{C}}\right)$.

Lemma 1. If $f \in M_{k}(\Gamma)$ then $\triangle_{k} f=\lambda f$, where $\lambda=\frac{k}{2}\left(1-\frac{k}{2}\right)$.

Proof Let $F=\sigma_{k}\left(y^{k / 2} f\right) \in \mathcal{C}_{k}(\Gamma \backslash G)$. It is enough to show that $\Delta F=\lambda F$. We have $H F=k F$ while $L F=0$. Thus using the second expression in (6)

$$
-4 \Delta F=\left(H^{2}-2 H+4 R L\right)=\left(k^{2}-2 k\right) F,
$$

and the statement follows. 
Theorem 1. Let $r \geqslant 0$ and let $f \in M_{-r}(\Gamma)$. Then the $r+1$-st derivative $f^{(r+1)} \in M_{r+2}(\Gamma)$.

This is Conjecture 1 when $n=1$. It was already proved in the introduction by another method.

Proof It is clear a priori from (7) that $f_{r+2}=\mathcal{R}_{r} \circ \ldots \circ \mathcal{R}_{-r+2} \circ \mathcal{R}_{-r}(f)$ is a linear combination of terms of the form $y^{-(r+1-i)} f^{(i)}$ where $0 \leqslant i \leqslant r+1$, and the coefficient of $f^{(r+1)}$ is $(2 i)^{r+1}$. If we can show that this function is holomorphic, it will follow that $y^{-(r+1-i)} f^{(i)}$ with $i>0$ have zero coefficient, hence

$$
(2 i)^{r+1} f^{(r+1)}=f_{r+2} \in \mathcal{M}_{r+2}(\Gamma \backslash \mathcal{H}) .
$$

The statement will therefore follow. We will prove this by computations in $U\left(\mathfrak{g}_{\mathbb{C}}\right)$, so it will be useful to transfer the function to the group. Let $F=\sigma_{-r}\left(y^{-r / 2} f\right) \in \mathcal{C}_{-r}(\Gamma \backslash G)$. By Lemma 1 we have $\Delta F=-\frac{r}{2}\left(1+\frac{r}{2}\right) F$. Since $\Delta$ commutes with $R$, we have

$$
\Delta F_{r}=-\frac{r}{2}\left(1+\frac{r}{2}\right) F_{r}, \quad F_{r}=R^{r} F .
$$

Also $H F_{r}=r F_{r}$ since $F_{r} \in \mathcal{C}_{r}(\Gamma \backslash G)$. Now using the first expression in (6) this means that

$$
r(r+2) F_{r}=-4 \Delta F_{r}=\left(H^{2}+2 H+4 L R\right) F_{r}=\left(r^{2}+2 r\right) F_{r}+4 L R F_{r} .
$$

It follows that $L R^{r+1} F=L R F_{r}=0$. Transferring this back to a statement about $f$, we see that

$$
\mathcal{L}_{r+2}\left(\mathcal{R}_{r} \circ \ldots \circ \mathcal{R}_{-r+2} \circ \mathcal{R}_{-r}(f)\right)=0,
$$

so $f_{r+2}$ is holomorphic, as required.

We now reinterpret this proof in terms of representations of $G=\mathrm{SL}_{2}(\mathbb{R})$. We will exhibit an indecomposable representation $\rho_{r}$ of $G$ (actually a $(\mathfrak{g}, K)$ module) which contains two "holomorphic vectors," one of weight $-r$ and one of weight $r+2$, corresponding to $f$ and $f^{(r+1)}$. Then we will show how, given a modular form of weight $-r$, one may construct a $(\mathfrak{g}, K)$-submodule of $C^{\infty}(\Gamma \backslash G)$ isomorphic to $\rho_{r}$.

Let $K=\mathrm{SO}(2)$, and let $(\pi, V)$ be a $(\mathfrak{g}, K)$-module. This means that we have compatible representations $\pi: K \longrightarrow \operatorname{End}(V)$ and $d \pi: \mathfrak{g} \longrightarrow \operatorname{End}(V)$. The compatibility amounts to the following condition. If $k \in \mathbb{Z}$ let

$$
V(k)=\left\{v \in V \mid \pi\left(\kappa_{\theta}\right) v=e^{i k \theta}\right\}, \quad \kappa_{\theta}=\left(\begin{array}{c}
\cos \theta \sin \theta \\
-\sin \theta \cos \theta
\end{array}\right) .
$$


It is assumed that $V$ is the algebraic direct sum of the $V(k)$, and that each $V(k)$ is finite-dimensional; and the compatibility of the representations $\pi$ and $d \pi$ amounts to the assumption that

$$
d \pi\left(\begin{array}{cc}
0 & 1 \\
-1 & 0
\end{array}\right) v=i k v, \quad v \in V(k) .
$$

We assume that $V$ is indecomposable, though not necessarily irreducible, and that each $V(k)$ is at most one-dimensional. The indecomposability implies that $\pi(-I)$ must operate by a scalar $(-1)^{\varepsilon}$. Thus $V(k)=0$ unless $k \equiv \varepsilon$ modulo 2 .

Let

$$
\hat{H}=\left(\begin{array}{cc}
1 & 0 \\
0 & -1
\end{array}\right), \quad \hat{R}=\left(\begin{array}{ll}
0 & 1 \\
0 & 0
\end{array}\right), \quad \hat{L}=\left(\begin{array}{ll}
0 & 0 \\
1 & 0
\end{array}\right) \in \mathfrak{g} .
$$

Then $H, R$ and $L$ defined by (5) are obtained by applying $\operatorname{Ad}\left(c^{-1}\right)$ to $\hat{H}$, $\hat{R}$ and $\hat{L}$, where

$$
c=\frac{1}{\sqrt{2 i}}\left(\begin{array}{cc}
1 & -i \\
1 & i
\end{array}\right), \quad c^{-1}=\frac{1}{\sqrt{2 i}}\left(\begin{array}{cc}
i & i \\
-1 & 1
\end{array}\right)
$$

denotes the Cayley transform (in $\operatorname{SL}(2, \mathbb{C})$ ). We may interpret (9) as the condition that $V(k)$ is the $k$-eigenspace of $H$. With this in mind, the commutation conditions $[H, R]=2 R$ and $[H, L]=-2 L$ imply that $L(V(k)) \subseteq$ $V(k-2)$ and $R(V(k)) \subseteq V(k+2)$. Also let $\Delta$ be the Casimir element of $U(\mathfrak{g})$, defined by

$$
-4 \Delta=\hat{H}^{2}+2 \hat{R} \hat{L}+2 \hat{L} \hat{R}=H^{2}+2 R L+2 L R .
$$

The center of $U(\mathfrak{g})$ is $\mathbb{C}[\Delta]$. It is easy to see that the center of $U(\mathfrak{g})$ must act by scalars on indecomposable admissible $(\mathfrak{g}, K)$-modules; this is a version of Schur's Lemma. So $\Delta$ acts by a scalar value $\lambda$ on $V$.

We call $v \in V(k)$ a holomorphic vector if $v \neq 0$ and $\pi(L) v=0$. If $V$ is irreducible, then $V$ can have at most one holomorphic vector. The irreducible $(\mathfrak{g}, K)$-modules of $\mathrm{SL}(2, \mathbb{R})$ that have holomorphic vectors are the finite-dimensional representations, the holomorphic discrete series and the holomorphic weight one "limit of discrete series."

Let us recall how the discrete series representations are embedded in the principal series. Let $s$ be a complex number, and let $\varepsilon=0$ or 1 . Let $\chi_{s, \varepsilon}$ denote the character

$$
\chi_{s, \varepsilon}\left(\begin{array}{c}
y^{1 / 2} x y^{-1 / 2} \\
y^{-1 / 2}
\end{array}\right)=\operatorname{sgn}(y)^{\varepsilon}|y|^{s} .
$$


Let $\operatorname{Ind}\left(\chi_{s, \varepsilon}\right)$ denote the $(\mathfrak{g}, K)$-module obtained by non-normalized induction. Thus $V(k)$ is zero unless $k \equiv \varepsilon$ modulo 2 , in which case it is onedimensional, and spanned by $v_{k}=v_{k, s, \varepsilon}$, where

$$
v_{k, s, \varepsilon}\left(\left(\begin{array}{c}
y^{1 / 2} x y^{-1 / 2} \\
y^{-1 / 2}
\end{array}\right) \kappa_{\theta}\right)=\operatorname{sgn}(y)^{\varepsilon}|y|^{s} e^{i k \theta} .
$$

Proposition 1. We have

$$
d \pi(L) v_{k}=\frac{1}{2}(2 s-k) v_{k-2}, \quad d \pi(R) v_{k}=\frac{1}{2}(2 s+k) v_{k+2}
$$

Proof It follows from the fact that $[H, L]=-2 L$ and $[H, R]=2 R$, and from the fact that $v_{k}$ spans the $k$-eigenspace $V(k)$ of $H$ that $L v_{k} \in V(k-2)$ and $R v_{k} \in V(k+2)$. Thus it is sufficient to compute the values of $L v_{k}$ and $R v_{k}$ at the identity. We first show

$$
d \pi(\hat{H}) v_{k}(I)=2 s, \quad d \pi(\hat{R}) v_{k}(I)=0, \quad d \pi(\hat{L}) v_{k}(I)=-i k .
$$

Indeed,

$$
\begin{gathered}
d \pi(\hat{H}) v_{k}(I)=\left.\frac{d}{d t} \pi(\exp (t \hat{H})) v_{k}(I)\right|_{t=0}=\left.\frac{d}{d t} v_{k}\left(\begin{array}{c}
e^{t} \\
e^{-t}
\end{array}\right)\right|_{t=0}=\left.\frac{d}{d t} e^{2 t s}\right|_{t=0}=2 s, \\
d \pi(\hat{R}) v_{k}(I)=\left.\frac{d}{d t} \pi(\exp (t \hat{R})) v_{k}(I)\right|_{t=0}=\left.\frac{d}{d t} v_{k}\left(\begin{array}{r}
1 t \\
1
\end{array}\right)\right|_{t=0}=\left.\frac{d}{d t} 1\right|_{t=0}=0
\end{gathered}
$$

and since $\hat{R}-\hat{L}=i H, d \pi(\hat{L}) v_{k}(I)=-d \pi(\hat{R}-\hat{L}) v_{k}=-i d \pi(H) v_{k}(I)=-i k$. Now

$$
\begin{aligned}
& d \pi(R) v_{k}(1)=\frac{1}{2} d \pi(\hat{H}+i \hat{R}+i \hat{L}) v_{k}(1)=\frac{1}{2}(2 s+k), \\
& d \pi(L) v_{k}(1)=\frac{1}{2} d \pi(\hat{H}-i \hat{R}-i \hat{L}) v_{k}(1)=\frac{1}{2}(2 s-k) .
\end{aligned}
$$

Proposition 2. The eigenvalue of $\Delta$ on $\operatorname{Ind}_{s, \varepsilon}$ is $s(1-s)$.

Proof It follows easily from (10) and Proposition 1 that $\Delta$, applied to any $v_{k}$ multiplies it by this constant.

The principal series representation $\operatorname{Ind}\left(\chi_{s, \varepsilon}\right)$ is reducible if $s=\frac{r}{2}$ where $r$ is an integer congruent to $\varepsilon$ modulo 2. There are two cases, depending on 
whether $r$ is positive or negative. If $r>0$, then $L v_{r}=0$ and $R v_{-r}=0$. This means that $V$ has two invariant subspaces

$$
D_{r}^{+}=\bigoplus_{\substack{k \geqslant r \\ k \equiv \varepsilon \bmod 2}} V(k), \quad D_{r}^{-}=\bigoplus_{\substack{k \leqslant-r \\ k \equiv \varepsilon \bmod 2}} V(k) .
$$

These are closed under $H, R$ and $L$ and so they are $(\mathfrak{g}, K)$-submodules. The quotient

$$
\operatorname{Ind}\left(\chi_{r / 2, \varepsilon}\right) /\left(D_{r}^{+} \oplus D_{r}^{-}\right)
$$

is finite dimensional - in fact, its dimension is $r-2$, and it is spanned by the images of the $V(k)$ with $2-r \leqslant k \leqslant r-2$. The space $D_{r}^{+}$has a holomorphic vector $v_{r}$, and this is a representation of the holomorphic discrete series provided $r \geqslant 2$. (If $r=1$ it is a "limit of discrete series.")

If $r$ is negative and $\varepsilon \equiv r \bmod 2$, then $\operatorname{Ind}\left(\chi_{r / 2, \varepsilon}\right)$ is again reducible. However it has the same composition factors as $\operatorname{Ind}\left(\chi_{(2-r) / 2, \varepsilon}\right)$, namely the two discrete series and the $r-2$-dimensional representation. There is an important distinction: $D_{r}^{+}$and $D_{r}^{-}$appear as quotients rather than subrepresentations of $\operatorname{Ind}\left(\chi_{(2-r) / 2, \varepsilon}\right)$.

Now we may construct an indecomposible representation with two holomorphic vectors. Let $r>0$, and let $\varepsilon=0$ or 1 be congruent to $r$ modulo 2 . Consider the quotient

$$
\rho_{r}=\operatorname{Ind}\left(\chi_{(r+2) / 2, \varepsilon}\right) / D_{r+2}^{-} .
$$

Let $u_{k}$ denote the image of $v_{k}$ in this representation. Then $u_{-r}$ and $u_{r+2}$ are both holomorphic vectors. The space on which it acts is

$$
V_{\rho}=\bigoplus_{\substack{k \geqslant-r \\ k \equiv r \bmod 2}} V_{\rho}(k), \quad V_{\rho}(k)=\mathbb{C} u_{k} .
$$

The Lie algebra acts by the rules

$$
\begin{aligned}
d \rho(L) u_{k} & = \begin{cases}\frac{1}{2}(r+2-k) v_{k-2} & \text { if } k \geqslant-r \\
0 & \text { if } k=-r\end{cases} \\
d \rho(R) u_{k} & =\frac{1}{2}(r+2+k) v_{k+2} \\
d \rho(H) u_{k} & =k u_{k}
\end{aligned}
$$

The eigenvalue of $\Delta$ is $-\frac{r}{2}\left(1+\frac{r}{2}\right)$.

Proposition 3. Let $r>0$ and let $f \in M_{-r}(\Gamma)$. Then $\sigma_{-r}\left(y^{-r / 2} f\right) \in C^{\infty}(G)$ generates a $(\mathfrak{g}, K)$-module isomorphic to $\rho_{r+2}$. 
Proof Define $f_{-r}=\sigma_{-r}\left(y^{-r / 2} f\right)$ and, recursively, for $k \geqslant-r, k \equiv r \bmod$ 2

$$
f_{k+2}=\frac{2}{r+k+2} R f_{k} .
$$

It may be easily checked that $\rho_{k} \longmapsto f_{k}$ is an isomorphism of $V_{\rho}$ onto the span of the $f_{k}$, with $k \geqslant-r, k \equiv r \bmod 2$.

\section{MAass OPERATORS FOR $\operatorname{Sp}(2 n)$}

In this section we review Maass operators for the symplectic group, and their origin in the Lie algebra. See Maass [13], [11] and [12] and Harris [8].

Let $G=\operatorname{Sp}(2 n, \mathbb{R}), G_{\mathbb{C}}=\operatorname{Sp}(2 n, \mathbb{C})$, and let $\mathfrak{g}, \mathfrak{g}_{\mathbb{C}}$ be their Lie algebras. The Cayley transform $c \in \operatorname{Sp}(2 n, \mathbb{C})$ is defined by

$$
c=\frac{1}{\sqrt{2 i}}\left(\begin{array}{cc}
I_{n}-i I_{n} \\
I_{n} & i I_{n}
\end{array}\right), \quad c^{-1}=\frac{1}{\sqrt{2 i}}\left(\begin{array}{cc}
i I_{n} & i I_{n} \\
-I_{n} & I_{n}
\end{array}\right) .
$$

The map

$$
A+i B \longmapsto\left(\begin{array}{cc}
A & B \\
-B & A
\end{array}\right)
$$

embeds $U(n)$ into $\operatorname{Sp}(2 n, \mathbb{R})$, and is easily checked to be a homomorphism. Let $K$ be the image of this map. We have

$$
c K c^{-1}=\left\{\left(\begin{array}{cc}
A+i B & \\
& A-i B
\end{array}\right) \mid A+i B \in U(n)\right\} .
$$

Thus $\operatorname{Ad}(c)$ is the differential of an inner automorphism of $\operatorname{Sp}(2 n, \mathbb{C})$ that takes $K$ into the Levi factor $M U$ of the parabolic subgroup

$$
\begin{aligned}
& P=M U, \quad M \cong \operatorname{GL}(n, \mathbb{C})=\left\{\left(\begin{array}{l}
g \\
{ }^{t} g^{-1}
\end{array}\right) \mid g \in \mathrm{GL}(n, \mathbb{C})\right\}, \\
& U=\left\{\left(\begin{array}{c}
I X \\
I
\end{array}\right) \mid X={ }^{t} X\right\} \text {. }
\end{aligned}
$$

If $X \in \operatorname{Mat}_{n}(\mathbb{C})$ we will denote

$$
\hat{H}_{X}=\left(\begin{array}{ll}
X & \\
& -{ }^{t} X
\end{array}\right) \in \mathfrak{g}_{\mathbb{C}}, \quad H_{X}=\operatorname{Ad}\left(c^{-1}\right) \hat{H}_{X},
$$

and if $X$ is symmetric, we will also denote

$$
\begin{array}{cc}
\hat{R}_{X}=\left(\begin{array}{cc}
0 & X \\
0 & 0
\end{array}\right), & \hat{L}_{X}=\left(\begin{array}{cc}
0 & 0 \\
X & 0
\end{array}\right), \\
R_{X}=\operatorname{Ad}\left(c^{-1}\right) \hat{R}_{X}, & L_{X}=\operatorname{Ad}\left(c^{-1}\right) \hat{L}_{X} .
\end{array}
$$


We recall that the irreducible representations of $U(n)$ are parametrized by decreasing sequences of integers

$$
\lambda=\left(\lambda_{1}, \lambda_{2}, \cdots, \lambda_{n}\right), \quad \lambda_{1} \geqslant \lambda_{2} \geqslant \cdots \geqslant \lambda_{n} .
$$

In this parametrization, the representation $\pi_{\lambda}$ corresponding to $\lambda$ has highest weight vector $\lambda$, which we identify with the rational character

$$
\left(\begin{array}{ccc}
t_{1} & & \\
& \ddots & \\
& & t_{n}
\end{array}\right) \longmapsto \prod_{i=1}^{n} t_{i}^{\lambda_{i}}
$$

of the diagonal torus. In particular if $\lambda=(k, \cdots, k)$ then $\pi_{\lambda}$ is the one dimensional representation with character $\operatorname{det}^{k}$. If the $\lambda_{i}$ are nonnegative we describe $\lambda$ as a partition (of length $\leqslant n$ ) and if furthermore the $\lambda_{i}$ are even we describe $\lambda$ as even.

We are interested in representations of the metaplectic group, that is, the double cover of $\operatorname{Sp}(2 n, \mathbb{R})$. This is the unique nontrivial central extension:

$$
1 \longrightarrow \mu_{2} \longrightarrow \widetilde{\mathrm{Sp}}(2 n, \mathbb{R}) \longrightarrow \mathrm{Sp}(2 n, \mathbb{R}) \longrightarrow 1,
$$

where $\mu_{2}$ is a group of order two.

Let $\tilde{K}$ be the preimage of $K=U(n)$ in $\widetilde{\operatorname{Sp}}(2 n, \mathbb{R})$. As we will now explain, the irreducible representations of $\tilde{K}$ may be parametrized by decreasing sequences

$\lambda=\left(\lambda_{1}, \lambda_{2}, \cdots, \lambda_{n}\right), \quad \lambda_{i} \in \frac{1}{2} \mathbb{Z}, \quad \lambda_{1} \geqslant \lambda_{2} \geqslant \cdots \geqslant \lambda_{n}, \quad \lambda_{i} \equiv \lambda_{j} \bmod \mathbb{Z}$.

The fundamental group $\pi_{1}(K) \cong \mathbb{Z}$, and so $K$ has a unique nontrivial double cover, which is easily described. Indeed, we may identify $\tilde{K}$ with the group $\left\{(g, t) \mid g \in U(n), t \in \mathbb{C}^{\times}, t^{2}=\operatorname{det}(g)\right\}$. We naturally denote the character $(g, t) \longmapsto t$ of $\tilde{K}$ by $\operatorname{det}^{1 / 2}$. Now if $\pi$ is an irreducible representation of $\tilde{K}$ which factors through $K$, then we denote it as $\pi_{\lambda}$, where $\lambda$ is the highest weight vector of the corresponding representation of $K$, identified with an integer sequence. Otherwise, it is of the form $\pi_{\mu} \otimes \operatorname{det}^{1 / 2}$, where $\mu$ is an integer sequence, in which case we denote the representation $\pi_{\lambda}$ with

$$
\lambda=\left(\mu_{1}+\frac{1}{2}, \cdots, \mu_{n}+\frac{1}{2}\right) .
$$

The Lie algebra of $\tilde{K}$ is the same as the Lie algebra of $K=U(n)$, and is generated by $-i H_{X}$ where $X \in \operatorname{Mat}_{n}(\mathbb{C})$ is skew-Hermitian, and $H_{X}=$ $\operatorname{Ad}\left(c^{-1}\right) \hat{H}_{X}$. 
Let $(\pi, V)$ be a $(\mathfrak{g}, \tilde{K})$-module. If $0 \neq v \in V$ we call $v$ a semispherical vector if $\mathbb{C} v$ is stable under $K$. In this case $\pi(g) v=\operatorname{det}(g)^{k} v$ when $g \in \tilde{K}$ for some $k \in \frac{1}{2} \mathbb{Z}$, and we call $k$ the weight of $v$. We call $v$ a holomorphic vector if it is semispherical, and if $\pi\left(L_{X}\right) v=0$ for all symmetric $X$.

The $R_{X}$, where $X \in \operatorname{Mat}_{n}(\mathbb{C})$ are symmetric are an abelian complex Lie subalgebra $\mathfrak{R}$ of $\mathfrak{g}_{\mathbb{C}}$. We may therefore identify the universal enveloping algebra $U(\mathfrak{R})$ and the symmetric algebra $S(\mathfrak{R})$.

Proposition 4. Let $v$ be a holomorphic vector of weight $k$ in $V$. Then $S(\mathfrak{R}) v$ is an invariant subspace. If $\pi_{\lambda}$ is a representation of $\tilde{K}$ that occurs in the decomposition of $S(\Re) v$ over $\tilde{K}$, then $\left(\lambda_{1}-k, \cdots, \lambda_{n}-k\right)$ is an even partition. The representation of $\tilde{K}$ on $S(\mathfrak{R}) v$ is multiplicity-free.

Compare Harris [8], Proposition 3.1.

Proof We note that in the adjoint representation $U(n)$ stabilizes

$$
\mathcal{R}=\left\{R_{X} \mid X \text { symmetric }\right\},
$$

and the action of $U(n)$ is equivalent to the action on symmetric matrices by

$$
U(n) \ni g \longmapsto g X^{t} g .
$$

That is, if $g=A+B i$ then

$$
\operatorname{Ad}\left(\begin{array}{cc}
A & B \\
-B & A
\end{array}\right) R_{X}=R_{g X^{t} g} .
$$

From this it follows that $S(\mathfrak{R}) v$ is invariant under $\tilde{K}$.

To check that it is invariant under $U\left(\mathfrak{g}_{\mathbb{C}}\right)$, we will show that $U\left(\mathfrak{g}_{\mathbb{C}}\right) v=$ $S(\mathfrak{R}) v$. We note that every element of $U\left(\mathfrak{g}_{\mathbb{C}}\right)$ can be written as a linear combination of elements of the form

$$
R_{X_{1}} \cdots R_{X_{p}} H_{Y_{1}} \cdots H_{Y_{q}} L_{Z_{1}} \cdots L_{Z_{s}} .
$$

Unless $s=0$, such an element kills $v$. If $s=0$, then $H_{Y_{1}} \cdots H_{Y_{q}} v$ is a constant multiple of $v$, and so $R_{X_{1}} \cdots R_{X_{p}} H_{Y_{1}} \cdots H_{Y_{q}} L_{Z_{1}} \cdots L_{Z_{s}} v \in S(\mathfrak{R}) v$, as required.

We have checked that $S(\mathfrak{R}) v$ is a $(\mathfrak{g}, \tilde{K})$-submodule of $V$. It is clear from (12) that the action of $\tilde{K}$ is by a quotient of $\operatorname{det}^{k} \otimes S(\mathcal{R})$. Now we claim that the action (11) is equivalent to the symmetric square action of $U(n)$ on $\operatorname{Sym}_{2}\left(\mathbb{C}^{n}\right)$. Indeed, an equivalence is given by

$$
v_{1} \vee v_{2} \longmapsto v_{1}^{t} v_{2}+v_{2}^{t} v_{1}
$$


where $v_{1}$ and $v_{2}$ are column vectors, so the right-hand side is a square matrix in $\mathcal{R}$.

The decomposition of $S(\mathcal{R})$ is well-known and essentially due to Littlewood [10]. See Bump [2] Theorem 46.1 or Goodman and Wallach [5] for a proof that

$$
S(\mathcal{R}) \cong \bigoplus_{\lambda \text { an even partition }} \pi_{\lambda}
$$

Since $S(\Re) \cong \operatorname{det}^{k} \otimes S(\mathcal{R})$ as $\tilde{K}$-modules, the statement follows.

The one-dimensional representations of $U(n)$ that occur in (13) are those of the form $\lambda=(2 l, \cdots, 2 l)$. Thus weights of the semispherical vectors that occur in $S(\mathfrak{R}) v$ are a subset of

$$
\{(2 l+k, \cdots, 2 l+k) \mid 0 \leqslant l \in \mathbb{Z}\} .
$$

If $\pi$ is infinite-dimensional these will all occur; for example, this is the case when $S(\mathfrak{R}) v$ is a representation of the holomorphic discrete series. However nothing in our assumptions preclude $V$ and hence $S(\Re) v$ from being finitedimensional.

The Maass operators which we now introduce shift between these semispherical vectors. If $1 \leqslant i, j \leqslant n$, let $E_{i j}$ be the square matrix with a 1 in the $i, j$ position and zeros elsewhere, and let $X_{i j}=E_{i j}+E_{j i}$. Let

$$
M_{+}=\operatorname{det}\left(R_{X_{i j}}\right) \in S(\Re) .
$$

Remark 1. The notation $\operatorname{det}\left(R_{X_{i j}}\right)$ is potentially ambiguous. We do not mean the determinant of the matrix $R_{X_{i j}}$. Rather we mean that we regard $R_{X_{i j}}$ as an element of the commutative ring $\mathcal{R}$, and we form the determinant of the matrix whose $i, j$ entry is $R_{X_{i j}}$.

For example if $n=2$, we will denote

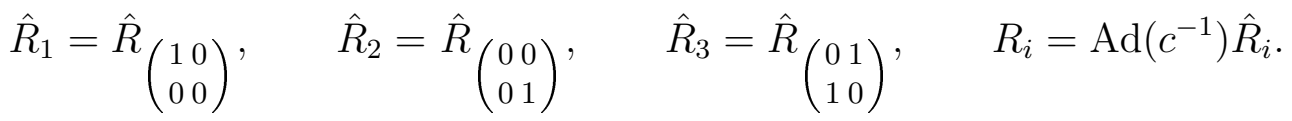

Then $R_{X_{11}}=2 R_{1}, R_{X_{12}}=R_{X_{21}}=R_{3}$ and $R_{X_{22}}=2 R_{2}$ so $M_{+}=4 R_{1} R_{2}-R_{3}^{2}$.

Lemma 2. Let $g \in \mathrm{GL}(n, \mathbb{C})$, acting on $\mathcal{R}$ and hence on $S(\mathcal{R})$ by $g: X \longmapsto$ $g X^{t} g$. Let $\mathcal{M}_{+}$be the element $\operatorname{det}\left(R_{i j}\right)$ of $S(\mathcal{R})$. Then $\mathcal{M}_{+}$is multiplied by $\operatorname{det}(g)^{2}$ in this action. 
Proof If $X \in \mathcal{R}$ let $p_{X}: \mathcal{R} \longrightarrow \mathbb{C}$ be defined by $p_{X}(Y)=\frac{1}{2} \operatorname{tr}(X Y)$. The map $X \longmapsto p_{X}$ extends to an isomorphism $\alpha$ of $S(\mathcal{R})$ onto the ring $P(\mathcal{R})$ of polynomial functions on $\mathcal{R}$. We let $U(n)$ act on $P(\mathcal{R})$ by $g f(X)=f\left({ }^{t} g X g\right)$. Then the map $X \longmapsto p_{X}$ is equivariant. We note that $\alpha\left(X_{i j}\right)$ is the $i, j$ coordinate function on $\mathcal{R}$, so $\alpha\left(\mathcal{M}_{+}\right)$is the determinant map $\mathcal{R} \longrightarrow \mathbb{C}$. The statement is now clear.

Proposition 5. Let $w$ be a semispherical vector of weight $l$ in a $(\mathfrak{g}, \tilde{K})$ module $V$. If $M_{+} w \neq 0$ then $M_{+} w$ is semispherical of weight $l+2$.

Proof Since $H_{X}$ with $X \in \operatorname{Mat}_{n}(\mathbb{C})$ span the complexified Lie algebra of $\tilde{K}$, the assumption that $w$ be a semispherical vector of weight $l$ amounts to the fact that $H_{X} w=l \operatorname{tr}(X) w$ for $H \in \operatorname{Mat}_{n}(\mathbb{C})$. Now

$$
H_{X} M_{+} w=\left[H_{X}, M_{+}\right] w+M_{+} H_{X} w=\left[H_{X}, M_{+}\right] w+l \operatorname{tr}(X) M_{+} w,
$$

so the assertion reduces to showing that $\left[H_{X}, M_{+}\right]=2 \operatorname{tr}(X) M_{+}$in $U\left(\mathfrak{g}_{\mathbb{C}}\right)$. This is a Lie algebra version of the assertion that $\operatorname{Ad}(g) M_{+}=\operatorname{det}(g)^{2} M_{+}$ when $g \in U(n)$. If $g=A+i B$ with $A$ and $B$ real, identified as usual with the symplectic matrix $\left(\begin{array}{cc}A & B \\ -B & A\end{array}\right)$, then $\operatorname{Ad}(g)$ acts by (12). Thus the statement follows from Lemma 2.

Thus if $v$ is a holomorphic vector, $M_{+}$shifts one-dimensional spaces of semispherical vectors in $S(\mathfrak{R}) v$, starting with $v$ itself, successively into each other. Similarly $M_{-}=\operatorname{det}\left(L_{X_{i j}}\right)$ shifts a semispherical vector of weight $l$ to a semispherical vector of weight $l-2$.

Conjecture 2. Let $r \geqslant 0$ be an integer. If $v$ is a holomorphic vector of weight $-r+\frac{n-1}{2}$ in a $(\mathfrak{g}, \tilde{K})$-module of $\widetilde{\operatorname{Sp}}(2 n, \mathbb{R})$, then $M_{+}^{r+1} v$ is a holomorphic vector of weight $r+2+\frac{n-1}{2}$.

\section{Modular Forms}

Theorem 2. Conjecture 2 implies Conjecture 1.

Proof Let $S$ be an analytic subset of $\Gamma \backslash \mathcal{H}_{n}$ of codimension one where the modular form $f$ will be allowed to be polar. Let $\mathcal{H}_{n}^{S}$ be the preimage of $\mathcal{H}_{n}-S$ in $\mathcal{H}_{n}$, which is an open set, and let $G^{S}$ be the preimage of $\mathcal{H}_{n}^{S}$ under the map $g \longmapsto g\left(i I_{n}\right)=(A i+B)(C i+D)^{-1}, g=\left(\begin{array}{c}A B \\ C D\end{array}\right) \in G$. If 
$f \in C^{\infty}\left(\mathcal{H}_{n}^{S}\right)$ denote

$$
\left(\left.f\right|_{k} g\right)(Z)=j_{k}(g, Z) f(g Z),
$$

where $j_{k}$ is as in the introduction.

We assume first that $k$ is integral, and discuss the modification needed when $k$ is half-integral afterwards. Let $\mathcal{M}_{k}^{S}(\Gamma, \chi)$ be the space of smooth functions on $\mathcal{H}_{n}^{S}$ satisfying (3). They are not assumed to be holomorphic. The subspace of holomorphic functions will be denoted $M_{k}^{S}(\Gamma, \chi)$. Denote by $\mathcal{C}_{k}\left(\Gamma \backslash \mathcal{H}_{n}^{S}, \chi\right)$ the space of smooth functions $f: \mathcal{H}_{n}^{S} \longrightarrow \mathbb{C}$ such that

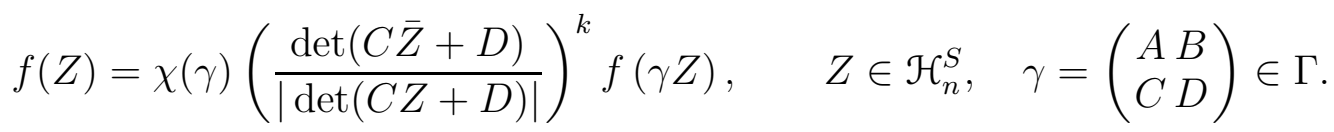

Also, let $\mathcal{C}_{k}\left(\Gamma \backslash G^{S}, \chi\right)$ be the space of smooth functions $f: G^{S} \longrightarrow \mathbb{C}$ such that $f(g)=\chi(\gamma) f(\gamma g)$ for $\gamma \in \Gamma$ and

$$
f\left(g\left(\begin{array}{cc}
A & B \\
-B & A
\end{array}\right)\right)=\operatorname{det}(\kappa)^{k} f(g), \quad \kappa=A+B i \in U(n) .
$$

If $k$ is half-integral, we modify these definitions as follows. The condition (1) implicitly assumes a choice of square root. We ask that a choice of square root be made in the function

$$
(g, Z) \longmapsto J_{k}(g, Z)=\operatorname{det}(C Z+D)^{-k}, \quad g=\left(\begin{array}{ll}
A & B \\
C & D
\end{array}\right) \in \operatorname{Sp}(2 n, \mathbb{R})
$$

which is continuous as a function of $Z$. (It is not possible to make it continuous of $g$.) Then

$$
\sigma\left(g_{1}, g_{2}\right)=\frac{J_{k}\left(g_{1} g_{2}, Z\right)}{J_{k}\left(g_{1}, g_{2} Z\right) J_{k}\left(g_{2}, Z\right)} \in\{ \pm 1\}
$$

is constant as a function of $Z$, and satisfies the cocycle relation

$$
\sigma\left(g_{1}, g_{2} g_{3}\right) \sigma\left(g_{2}, g_{3}\right)=\sigma\left(g_{1} g_{2}, g_{3}\right) \sigma\left(g_{1}, g_{2}\right) .
$$

Hence this is a 2-cocycle in $H^{2}(G,\{ \pm 1\})$ determining a double cover $\tilde{G}=$ $\widetilde{\mathrm{Sp}}(2 n, \mathbb{R})$. It is the same group considered in Section 2. Elements of $\tilde{G}$ are pairs $(g, \varepsilon)$ with $g \in G$ and $\varepsilon= \pm 1$, and the multiplication is given by

$$
\left(g_{1}, \varepsilon_{1}\right)\left(g_{2}, \varepsilon_{2}\right)=\left(g_{1} g_{2}, \sigma\left(g_{1}, g_{2}\right) \varepsilon_{1} \varepsilon_{2}\right) .
$$

The cocycle relation (2) means that

$$
\tilde{\chi}(\gamma, \varepsilon)=\chi(\gamma) \varepsilon
$$

is a character of the preimage $\tilde{\Gamma}$ of $\Gamma$ in $\tilde{G}$. 
Now let $\tilde{G}^{S}$ be the preimage of $G^{S}$ in $\tilde{G}$. Whether $k$ is integral or halfintegral we have maps

$$
\mathcal{M}_{k}^{S}(\Gamma, \chi) \stackrel{\operatorname{det}(Y)^{k / 2}}{\longrightarrow} \mathcal{C}_{k}^{S}\left(\Gamma \backslash \mathcal{H}_{n}, \chi\right) \stackrel{\sigma_{k}}{\longrightarrow} \mathcal{C}_{k}\left(\tilde{\Gamma} \backslash \tilde{G}^{S}, \chi\right)
$$

The first map is multiplication by $\operatorname{det}(Y)^{k / 2}$, where $Y=\operatorname{im}(Z)$. The second map $\sigma_{k}$ is defined by

$$
\sigma_{k}(f)(\tilde{g})=\left(\left.f\right|_{k} \tilde{g}\right)(i)
$$

where if

$$
\tilde{g}=\left(\left(\begin{array}{cc}
A & B \\
C & D
\end{array}\right), \varepsilon\right)
$$

we define

$$
\left(\left.f\right|_{k} \tilde{g}\right)(z)=\varepsilon \frac{\overline{J_{k}(g, Z)}}{\left|J_{k}(g, Z)\right|} f\left(\frac{A Z+B}{C Z+D}\right) .
$$

As in the introduction, we can use the exact sequence (14) to transfer the actions of $\mathfrak{g}$ and $\tilde{K}$ to actions on $\mathcal{M}_{k}^{S}(\Gamma, \chi)$. Particularly if $X$ is symmetric we have a commutative diagram

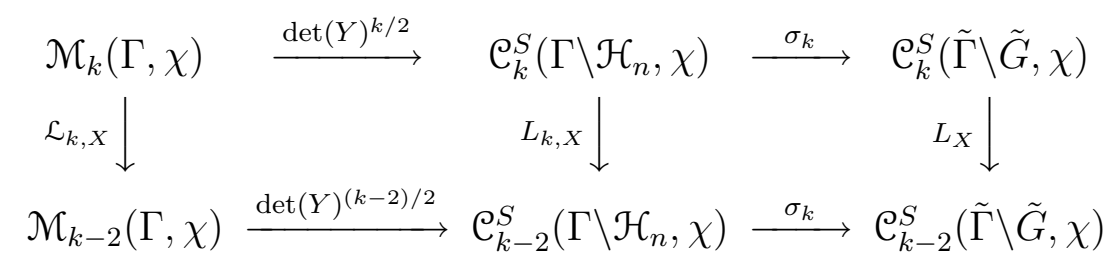

where the operator $\mathcal{L}_{k, X}$ is determined by the commutativity of the diagram. The operators $\mathcal{L}_{k, X}$ are made explicit in Harris [8], Section 2.3.1, and they are linear combinations of $\partial / \partial \bar{Z}_{i j}$ where $Z_{i j}$ are the matrix coefficients of $Z$. Thus as in [8], $\mathcal{L}_{k, X} f=0$ for all $X$ if and only if $f$ is holomorphic. Thus $f$ is holomorphic if and only if its image in $\mathrm{e}_{k}^{S}(\tilde{\Gamma} \backslash \tilde{G}, \chi)$ is a holomorphic vector $v$ in the $(\mathfrak{g}, \tilde{K})$-module it generates.

Similarly there are operators $\mathcal{R}_{k, X}$ determined by the commutativity of

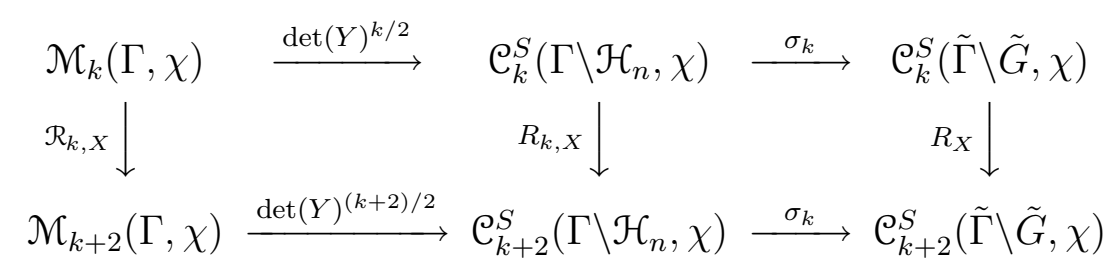

these too are made explicitly in Harris [8], Section 1.5.1. If $X_{i j}=E_{i j}+E_{j i}$, where $E_{i j}$ is the elementary matrix with 1 in the $i, j$ position and zeros 
elsewhere, then

$$
R_{k, X_{i j}}=(\operatorname{det} Y)^{\frac{n+1-k}{2}} \frac{\partial}{\partial Z_{i j}}(\operatorname{det} Y)^{\frac{k-n+1}{2}} .
$$

and

$$
\mathcal{R}_{k, X_{i j}}=(\operatorname{det} Y)^{\frac{n-1}{2}-k} \frac{\partial}{\partial Z_{i j}}(\operatorname{det} Y)^{-\frac{n-1}{2}+k},
$$

It is clear that applying

$$
\mathbb{M}_{k}=\operatorname{det}\left(\mathcal{R}_{k, X_{i j}}\right)
$$

to $f$ gives a linear combination of terms, one of which is $2^{n} \mathbb{D}_{n}^{r+1} f$, and the others are combinations of products of lower order derivatives of $f$ times various minors of $Y$. The only way this can be holomorphic is if all these other terms cancel. However the image of $\mathbb{M}_{k} f$ in $C_{r+2+\frac{n-1}{2}}^{S}(\tilde{\Gamma} \backslash \tilde{G}, \chi)$ is precisely $M_{+}^{r+1} v$, which by Conjecture 2 is a holomorphic vector. Hence the nonholomorphic terms of $\mathbb{M}_{k} f$ must cancel, and $2^{n} \mathbb{D}_{n}^{r+1} f=\mathbb{M}_{k} f$. We have excluded the set $S$ from these considerations but this is no problem since $\mathbb{D}_{n}^{r+1} f$ is a derivative of a meromorphic function, hence meromorphic.

\section{Computations in $\operatorname{Sp}(4, \mathbb{R})$}

Since $\operatorname{Sp}(4, \mathbb{R})$ has real rank equal to its complex rank, the ring of invariant differential operators on $G$ may be identified with the ring of invariant differential operators on its homogeneous space $\mathcal{H}_{2}$, or with the center $\mathcal{Z}$ of its universal enveloping algebra. Let $\mathfrak{g}=\mathfrak{s p}(4, \mathbb{R})$ and if $X \in \mathrm{Mat}_{2}(\mathbb{C})$ let

$$
\hat{H}_{X}=\left(\begin{array}{ll}
X & \\
& -{ }^{t} X
\end{array}\right) \in \mathfrak{g}_{\mathbb{C}}=\mathfrak{s p}(4, \mathbb{C}) .
$$

If $X$ is symmetric, let

$$
\hat{R}_{X}=\left(\begin{array}{cc}
0 & X \\
0 & 0
\end{array}\right), \quad \hat{L}_{X}=\left(\begin{array}{cc}
0 & 0 \\
X & 0
\end{array}\right) .
$$

Let

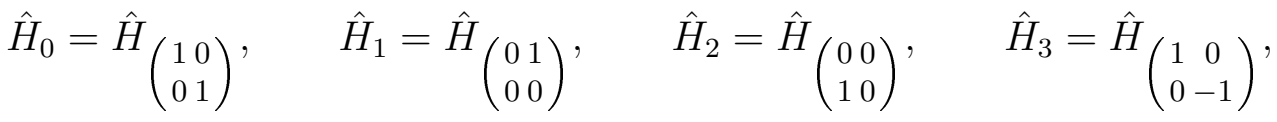

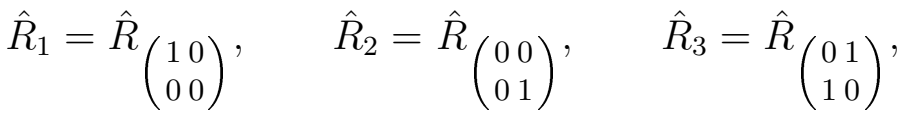

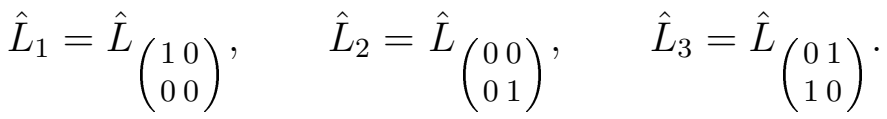


We also use the same notation without the "hats" for the corresponding Lie group elements obtained by applying $\operatorname{Ad}\left(c^{-1}\right)$. Thus

$$
H_{i}=\operatorname{Ad}\left(c^{-1}\right) \hat{H}_{i}, \quad R_{i}=\operatorname{Ad}\left(c^{-1}\right) \hat{R}_{i}, \quad L_{i}=\operatorname{Ad}\left(c^{-1}\right) \hat{L}_{i} .
$$

Note that $H_{i} \in \mathbb{C} \otimes \operatorname{Lie}(K)$.

Let $S\left(\mathfrak{g}_{\mathbb{C}}\right)$ and $U\left(\mathfrak{g}_{\mathbb{C}}\right)$ denote the symmetric algebra and universal enveloping algebra, respectively, of $\mathfrak{g}_{\mathbb{C}}$. Let $\lambda: S\left(\mathfrak{g}_{\mathbb{C}}\right) \longrightarrow U\left(\mathfrak{g}_{\mathbb{C}}\right)$ denote the symmetrization map, defined by

$$
\lambda\left(X_{1} \cdots X_{d}\right)=\frac{1}{d !} \sum_{w \in S_{d}} X_{\sigma(1)} \cdots X_{\sigma(d)} .
$$

It is not a ring homomorphism.

Proposition 6. The center $z$ of $U\left(\mathfrak{g}_{\mathbb{C}}\right)$ is a polynomial ring with two generators, of degrees 2 and 4 , respectively. They are $\mathfrak{D}_{2}=\lambda\left(D_{2}\right)$ and $\mathfrak{D}_{4}=\lambda\left(D_{4}\right)$, where $D_{2}$ and $D_{4}$ are the following ad $(\mathfrak{g})$-invariant elements of $S\left(\mathfrak{g}_{\mathbb{C}}\right)$. Of degree 2 :

$$
D_{2}=H_{0}^{2}+4 H_{1} H_{2}+H_{3}^{2}+8 L_{1} R_{1}+8 L_{2} R_{2}+4 L_{3} R_{3},
$$

and of degree 4 :

$$
\begin{aligned}
D_{4}= & 4 H_{0}^{2} H_{1} H_{2}+H_{0}^{2} H_{3}^{2}+16 H_{1} H_{2} L_{1} R_{1}+8 H_{0} H_{3} L_{1} R_{1}-16 H_{2}^{2} L_{2} R_{1} \\
& +8 H_{0} H_{2} L_{3} R_{1}-8 H_{2} H_{3} L_{3} R_{1}+16 L_{1}^{2} R_{1}^{2}-16 H_{1}^{2} L_{1} R_{2} \\
& +16 H_{1} H_{2} L_{2} R_{2}-8 H_{0} H_{3} L_{2} R_{2}+8 H_{0} H_{1} L_{3} R_{2} \\
& +8 H_{1} H_{3} L_{3} R_{2}-32 L_{1} L_{2} R_{1} R_{2}+16 L_{3}^{2} R_{1} R_{2}+16 L_{2}^{2} R_{2}^{2} \\
& +8 H_{0} H_{1} L_{1} R_{3}-8 H_{1} H_{3} L_{1} R_{3}+8 H_{0} H_{2} L_{2} R_{3}+8 H_{2} H_{3} L_{2} R_{3} \\
& +4 H_{3}^{2} L_{3} R_{3}+16 L_{1} L_{3} R_{1} R_{3}+16 L_{2} L_{3} R_{2} R_{3}+16 L_{1} L_{2} R_{3}^{2}
\end{aligned}
$$

Proof According to a well-known theorem of Harish-Chandra (essentially Lemma 36 of [6], or see Helgason [9]), if $\mathfrak{g}_{\mathbb{C}}$ is a complex semisimple Lie algebra of rank $r$, the center $z$ of $U\left(\mathfrak{g}_{\mathbb{C}}\right)$ is isomorphic to the ring of invariants of the Weyl group $W$, which is a polynomial ring in $r$ variables by a theorem of Chevalley [3]. The degrees $d_{1}, \cdots, d_{r}$ can be computed by a theorem of Solomon [14], which says that if $e_{p}$ is the $p$-th elementary symmetric polynomial $(p \leqslant r)$ and if $m_{p}=d_{p}-1$ then $e_{p}\left(d_{1}, \cdots, d_{r}\right)$ is the number of Weyl group elements whose fixed points have codimension $p$ in the action of $W$ on $\mathbb{R}^{r}$. If $\mathfrak{g}_{\mathbb{C}}=\mathfrak{s p}(4, \mathbb{C})$ this means that $m_{1}+m_{2}=4$ while $m_{1} m_{2}=3$, so $m_{1}=1$ and $m_{2}=3$. Thus $d_{1}$ and $d_{2}$ are 2 and 4 , as stated. 
If $X \in \mathfrak{g}$ then $\operatorname{ad}(X): \mathfrak{g}_{\mathbb{C}} \longrightarrow \mathfrak{g}_{\mathbb{C}}$ extends to a derivation of $S\left(\mathfrak{g}_{\mathbb{C}}\right)$, given by

$$
\operatorname{ad}(X)\left(Y_{1} \cdots Y_{r}\right)=\sum_{i=0}^{r} Y_{1} \cdots Y_{i-1}\left(\operatorname{ad}(X) Y_{i}\right) Y_{i+1} \cdots Y_{r} .
$$

Applying $\lambda$ and making use of the fact that $\operatorname{ad}(X) Y=X Y-Y X$ in $U(\mathfrak{g})$ gives

$$
\frac{1}{r !} \sum_{\sigma \in S_{r}} \sum_{i=0}^{r} Y_{\sigma(1)} \cdots Y_{\sigma(i-1)}\left(X Y_{\sigma(i)}-Y_{\sigma(i)} X\right) Y_{\sigma(i+1)} \cdots Y_{\sigma(r)},
$$

so after cancellation

$$
\lambda\left(\operatorname{ad}(X)\left(Y_{1} \cdots Y_{r}\right)\right)=X \lambda\left(Y_{1} \cdots Y_{r}\right)-\lambda\left(Y_{1} \cdots Y_{r}\right) X
$$

Let $S\left(\mathfrak{g}_{\mathbb{C}}\right)^{\operatorname{ad}(\mathfrak{g})}=\left\{\alpha \in S\left(\mathfrak{g}_{\mathbb{C}}\right) \mid \operatorname{ad}(X) \alpha=0\right.$ for all $\left.X \in \mathfrak{g}\right\}$ be the space of $\operatorname{ad}(\mathfrak{g})$-invariants. Then (15) shows that $\lambda$ takes $S\left(\mathfrak{g}_{\mathbb{C}}\right)^{\operatorname{ad}(\mathfrak{g})}$ into $Z$.

Elements of $S\left(\mathfrak{g}_{\mathbb{C}}\right)^{\text {ad( }(\mathfrak{g})}$ can be computed using a computer algebra package such as Mathematica. There is little point in reproducing these computations here, but let us offer a word as to how they were done. One starts with a general polynomial $\mathrm{F}$ of given degree in a set of generators, which one can represent as functions of an independent variable $t$. The polynomial F may be taken to be homogeneous. Thus for $\mathfrak{s p}(4)$, there will be 10 variables, and 55 terms for a homogeneous polynomial of degree 2 or 715 for a homogeneous polynomial of degree 4 . Let $\mathrm{Y}$ be another element of the Lie algebra. Denoting the variables as X1 $[\mathrm{t}], \mathrm{X} 2[\mathrm{t}], \ldots$ one may then differentiate the polynomial $F[\mathrm{X} 1[\mathrm{t}], \mathrm{X} 2[\mathrm{t}], \ldots]$ with respect to $t$, then wherever a derivative $\mathrm{Xi}$ '[t] occurs, substitute the value of $[\mathrm{Y}, \mathrm{Xi}$ ]. This gives the value of ad (Y) applied to $F$. Setting this to zero gives a set of linear equations in the coefficients, and solving these gives the ad invariants. In the case at hand, one arrives at the two generators listed above. Clearly $\lambda\left(D_{2}\right)^{2}$ and $\lambda\left(D_{4}\right)$ are linearly independent since $\lambda\left(D_{4}\right)$ does not involve the monomial $H_{0}^{4}$, and since we know $\mathcal{Z}$ is a polynomial ring in two variables, with generators in these degrees, they must be generators.

Remark 2. We made use of the fact that $S\left(\mathfrak{g}_{\mathbb{C}}\right)^{\text {ad }} \subseteq \mathcal{Z}$. It was shown by Harish-Chandra [7], Corollary at the bottom of p. 192 that $\lambda: S\left(\mathfrak{g}_{\mathbb{C}}\right)^{\text {ad }} \longrightarrow$ $Z$ is a linear isomorphism, though we do not need the surjectivity of this map. (See Helgason [9], Theorem 4.3 on p. 270.) It is of course not a ring homomorphism.

Let $\mathcal{J}$ be the left ideal generated by $H_{1}, H_{2}, H_{3}, L_{1}, L_{2}$ and $L_{3}$. 
Theorem 3. We have

$$
\mathfrak{D}_{2} \equiv H_{0}^{2}-6 H_{0}, \quad \mathfrak{D}_{4} \equiv-2 H_{0}^{2}+12 H_{0} \quad \bmod \mathcal{J} .
$$

In particular $2 \mathfrak{D}_{2}+\mathfrak{D}_{4} \in \mathcal{J}$.

Proof The computation proceeds by examining each term in $D_{i}$, applying $\lambda$ to it, and writing it as a polynomial in $H_{0}$ modulo J. We omit the details, which can be most easily checked using a computer.

Corollary 1. If $v$ is a holomorphic vector of weight $k$, then $\pi\left(\mathfrak{D}_{2}\right)$ and $\pi\left(\mathfrak{D}_{4}\right)$ act as scalars on $S(\mathfrak{R}) v$, with eigenvalues $4\left(k^{2}-3 k\right)$ and $-8\left(k^{2}-3 k\right)$, respectively.

Proof This is because $\pi\left(H_{0}\right) v=2 k v$, while $\mathcal{J}$ annihilates $v$.

Now let $r$ be a positive integer, and let $\alpha=r^{2}+2 r-\frac{5}{4}$. Thus $\alpha$ is the value of $k^{2}-3 k$ when $k=-r+\frac{1}{2}$. The equation

$$
k^{2}-3 k=\alpha
$$

has two roots $k=r_{1}$ and $k=r_{2}$ such that $r_{1}+r_{2}=3$. Since one root $r_{1}=-r+\frac{1}{2}$, the other root is $r+\frac{5}{2}$. Thus if $v$ is a holomorphic vector of weight $-r+\frac{1}{2}$ and we apply $M_{+}$to this vector $r+1$ times, we expect to obtain another holomorphic vector.

Theorem 4. Conjecture 2 is true if $n=2$.

Proof Let $v$ be a holomorphic vector of weight $-r+\frac{1}{2}$ in a $(\mathfrak{g}, \tilde{K})$-module $V$, and let $w=M_{+}^{r+1} v$. We will show that $w$ is holomorphic.

The first step is to show that $M_{-} w=0$. Let $\mathcal{J}$ be the left ideal generated by $H_{1}, H_{2}$ and $H_{3}$ and $L_{1}, L_{2}, L_{3}$. Apply $\mathfrak{D}_{2}$ and $\mathfrak{D}_{4}$ to $v$. Since $\mathcal{J}$ annihilates $v$ and $H_{0}$ has eigenvalue $1-2 r$, by Theorem 3 we have $\mathfrak{D}_{2} v=\delta v$ and $\mathfrak{D}_{4} v=-2 \delta v$ where

$$
\delta=(1-2 r)^{2}-6(1-2 r)=4 r^{2}+8 r-5 .
$$

Now since $\mathfrak{D}_{2}$ and $\mathfrak{D}_{4}$ commute with $\mathfrak{g}_{\mathbb{C}}$, they have the same eigenvalues applied to any vector in the space.

Let $u=M_{+}^{r} v$, and let $\mathcal{J}$ be the left ideal generated by $H_{1}, H_{2}$ and $H_{3}$. Since $u$ is semispherical, it is annihilated by $\mathcal{J}$. A computer calculation shows that if $M_{+}=4 R_{1} R_{2}-R_{3}^{3}$ and $M_{-}=4 L_{1} L_{2}-L_{3}^{2}$ then

$$
\begin{array}{r}
16 M_{-} M_{+}+4 \mathfrak{D}_{4}-\mathfrak{D}_{2}^{2}+8 H_{0} \mathfrak{D}_{2}+2 H_{0}^{2} \mathfrak{D}_{2} \equiv \\
H_{0}^{4}+8 H_{0}^{3}+4 H_{0}^{2}-48 H_{0} \bmod \mathcal{J} .
\end{array}
$$


Thus both sides have the same effect on $u$. Since $M_{-} M_{+} u=M_{-} w$, and since $H_{0} u=h u$, with $h=2 r+1$, we have

$$
\begin{gathered}
16 M_{-} w-8 \delta w-\delta^{2} w+8 h \delta w+2 h^{2} \delta w= \\
\left(h^{4}+8 h^{3}+4 h^{2}-48 h\right) w .
\end{gathered}
$$

But

$$
-8 \delta-\delta^{2}+8 h \delta+2 h^{2} \delta=h^{4}+8 h^{3}+4 h^{2}-48 h .
$$

(Both equal $16 r^{2}+96 r^{3}+136 r^{2}-24 r-35$.) Therefore $M_{-} w=0$.

Now suppose that $w$ is not holomorphic. We have

$$
\begin{array}{llr}
{\left[H_{1}, L_{1}\right]=-L_{3},} & {\left[H_{1}, L_{2}\right]=0, \quad\left[H_{1}, L_{3}\right]=-2 L_{2},} \\
{\left[H_{2}, L_{1}\right]=0, \quad\left[H_{2}, L_{2}\right]=-L_{3},} & {\left[H_{2}, L_{3}\right]=-2 L_{1} .} \\
{\left[H_{3}, L_{1}\right]=-2 L_{1}, \quad\left[H_{3}, L_{2}\right]=2 L_{2},} & {\left[H_{3}, L_{3}\right]=0 .}
\end{array}
$$

Since $H_{i} w=0$ with $i=1,2,3$ this implies that

$$
\begin{array}{ll}
H_{1} L_{1} w & =-L_{3} w, \quad H_{1} L_{2} w=0, \quad H_{1} L_{3} w=-2 L_{2} w, \\
H_{2} L_{1} w & =0, \quad H_{2} L_{2} w=-L_{3} w, \quad H_{2} L_{3} w=-2 L_{1} w, \\
H_{3} L_{1} w & =-2 L_{1} w, \quad H_{3} L_{2} w=2 L_{2} w, \quad H_{3} L_{3} w=0 .
\end{array}
$$

Also

$H_{0} L_{1} w=(2 r+3) L_{1} w, \quad H_{0} L_{2} w=(2 r+3) L_{2} w, \quad H_{0} L_{3} w=(2 r+3) L_{3} w$.

Similarly using $H_{i} u=0$ with $i=1,2,3$ we can compute

$$
\begin{gathered}
H_{1} R_{1} u=0, \quad H_{1} R_{2} u=R_{3} u, \quad H_{1} R_{3} w=2 R_{1} u, \\
H_{2} R_{1} u=R_{3} u, \quad H_{2} R_{2} u=0, \quad H_{2} R_{3} u=2 R_{2} u, \\
H_{3} R_{1} u=2 R_{1} w, \quad H_{3} R_{2} u=-2 R_{2} w, \quad H_{3} R_{3} u=0,
\end{gathered}
$$

and we also have

$H_{0} R_{1} w=(2 r+3) R_{1} w, \quad H_{0} R_{2} w=(2 r+3) R_{2} w, \quad H_{0} L_{3} w=(2 r+3) R_{3} w$.

We recall that the $H_{i}$ span the complexified Lie algebra $\mathfrak{k}_{\mathbb{C}}$ of $\tilde{K}$, the double cover of $\mathrm{SU}(2)$. Since we are assuming that $L_{i} u$ are not all zero, we see that both $L_{i} u$ and $R_{i} w$ span isomorphic $\tilde{K}$-modules. By Proposition 4 the space $S(\mathfrak{R}) v$ is multiplicity-free over $\tilde{K}$, so these two sets of vectors span the same three-dimensional vector space. Moreover,

$$
L_{1} w \longmapsto R_{2} u, \quad L_{2} w \longmapsto R_{1} u, \quad L_{3} w \longmapsto-R_{3} u
$$


is an isomorphism with repect to $\mathfrak{k}_{\mathbb{C}}$, so by Schur's Lemma, this map is a constant multiple of the identity map. Thus there is a nonzero constant $c$ such that $c L_{1} w=R_{2} u, c L_{2} w=R_{1} u$ and $c L_{3} w=-R_{3} u$. This means that

$$
2 L_{1} R_{1} u+2 L_{2} R_{2} u+L_{3} R_{3} u=2 L_{1} L_{2} w+2 L_{2} L_{1} w-L_{3}^{2} w=M_{-} w=0 .
$$

We can write

$$
\mathfrak{D}_{2}=H_{0}^{2}+2 H_{1} H_{2}+2 H_{2} H_{1}+H_{3}^{2}+6 H_{0}+8 L_{1} R_{1}+8 L_{2} R_{2}+4 L_{3} R_{3} \text {. }
$$

We apply this to $u$, recalling that $\mathfrak{D}_{2}$ acts by the scalar $4 r^{2}+8 r-5$ on the entire space. We obtain

$$
\left(4 r^{2}+8 r-5\right) u=\left(H_{0}^{2}+2 H_{1} H_{2}+2 H_{2} H_{1}+H_{3}^{2}+6 H_{0}\right) u .
$$

Since $H_{0} u=(2 r+1) u$ while $H_{i} u=0$ when $i=1,2,3$, this means that

$$
\left(4 r^{2}+8 r-5\right) u=\left((2 r+1)^{2}+6(2 r+1)\right) u=\left(4 r^{2}+16 r+7\right) u
$$

Simplifying gives $8 r+12=0$. This is a contradiction since $r \geqslant 0$. This proves the holomorphicity of $w$.

\section{REFERENCES}

[1] G. Bol. Invarianten linearer Differentialgleichungen. Abh. Math. Sem. Univ. Hamburg, 16:1-28, 1949.

[2] Daniel Bump. Lie groups, volume 225 of Graduate Texts in Mathematics. SpringerVerlag, New York, 2004.

[3] Claude Chevalley. Invariants of finite groups generated by reflections. Amer. J. Math., 77:778-782, 1955.

[4] YoungJu Choie and Haesuk Kim. An analogy of Bol's result on Jacobi forms and Siegel modular forms. J. Math. Anal. Appl., 257(1):79-88, 2001.

[5] R. Goodman and N. Wallach. Representations and Invariants of the Classical Groups, volume 68 of Encyclopedia of Mathematics and its Applications. Cambridge University Press, Cambridge, 1998.

[6] Harish-Chandra. On some applications of the universal enveloping algebra of a semisimple Lie algebra. Trans. Amer. Math. Soc., 70:28-96, 1951.

[7] Harish-Chandra. Representations of a semisimple Lie group on a Banach space. I. Trans. Amer. Math. Soc., 75:185-243, 1953.

[8] Michael Harris. Special values of zeta functions attached to Siegel modular forms. Ann. Sci. École Norm. Sup. (4), 14(1):77-120, 1981.

[9] Sigurdur Helgason. Groups and geometric analysis, volume 83 of Mathematical Surveys and Monographs. American Mathematical Society, Providence, RI, 2000. Integral geometry, invariant differential operators, and spherical functions, Corrected reprint of the 1984 original.

[10] D. Littlewood. The Theory of Group Characters and Matrix Representations of Groups. Oxford University Press, New York, 1940. 
[11] Hans Maass. Die Differentialgleichungen in der Theorie der elliptischen Modulfunktionen. Math. Ann., 125:235-263 (1953), 1952.

[12] Hans Maass. Die Differentialgleichungen in der Theorie der Siegelschen Modulfunktionen. Math. Ann., 126:44-68, 1953.

[13] Hans Maass. Siegel's modular forms and Dirichlet series. Springer-Verlag, Berlin, 1971. Dedicated to the last great representative of a passing epoch. Carl Ludwig Siegel on the occasion of his seventy-fifth birthday, Lecture Notes in Mathematics, Vol. 216.

[14] Louis Solomon. Invariants of finite reflection groups. Nagoya Math. J., 22:57-64, 1963.

Daniel Bump

Department of Mathematics, Stanford University

Stanford CA 94305-2125 USA

E-mail: bump@math.Stanford.EDU

Young-Ju Choie

Department of Mathematics, Pohang University of

Science and Technology Pohang, 790-784, Korea 